\title{
Establishment of tight junctions between epithelial cells
}

(electron microscopy/electrophysiology/intercellular contacts/membranes/wound healing)

\author{
A. J. HUDSPETH* \\ Department of Neurobiology, Harvard Medical School, Boston, Massachusetts 02115
}

Communicated by Stephen W. Kuffler, April 22, 1975

\begin{abstract}
Epithelia serve as barriers to the diffusion of solutes between body compartments, and must do so despite the frequent loss of cells. When single cells are experimentally removed from the Necturus gallbladder epithelium, contiguous cells migrate to fill the defect within $30 \mathrm{~min}$. Electrophysiological measurements show that the local electrical resistance across the epithelium in the region of a wound returns to normal in the same period of time; electron microscopy demonstrates that tight junctions are formed concurrently. Physiologically functional and morphologically recognizable tight junctions can thus be established within 30 min, demonstrating a mechanism for the rapid restoration of epithelial integrity after cell loss.
\end{abstract}

A distinctive structural feature of most vertebrate epithelia is the tight junction, or zonula occludens, which joins every cell with its neighbors. Each such junction is generally located near the cells' apices, and consists of one to several circumferential bands of close contacts between the apposed plasma membranes, manifested as membrane fusions in conventional electron microscopy $(1,2)$ and as intramembrane strands in freeze-fracture preparations (3-5). The close membrane contacts in effect form a series of gaskets surrounding each cell, and thereby constitute the principal barrier to the movement of solutes across an epithelium by diffusion between cells (6). Since the regulation of fluid composition within body compartments is one of the primary functions of epithelia, the integrity of tight junctions is essential to normal epithelial function, and must be maintained despite the frequent loss of cells through ageing and trauma (7).

The present report documents the rapid formation of tight junctions between newly apposed epithelial cells. Gallbladders were dissected from freshly killed and pithed mudpuppies (Necturus maculosus) and cut into pieces roughly 5 mm square. Each piece was mounted on a glass slide, with the epithelial surface uppermost, secured at its corners with metal spring clips, and bathed in modified L-15 tissue culture solution (8). Preparations were viewed and photographed through Nomarski differential interference contrast optics with a modified Zeiss WL microscope equipped with a $\times 40$, water-immersion lens.

The formation of tight junctions was initiated by bringing epithelial cells into contact with each other through the removal of one to several intervening cells. A selected cell (Fig. 4) was damaged by traumatic impalement or pricking with a fine glass microelectrode. After minimal damage, a cell generally rounded up and left the epithelium within 20 min. The process could be accelerated by pulling the damaged cell from the epithelium with the microelectrode. In either case, the loss of a cell (or group of cells) reliably pro-

* Present address: Division of Biology, California Institute of Technology, Pasadena, Calif. 91125. voked a stereotyped response of the adjacent cells (Figs. 4-6). Within $5 \mathrm{~min}$, cells began to extend processes and to move into the lacuna (Fig. 5). The defect was usually grossly closed in $15 \mathrm{~min}$, and often undetectable after $30 \mathrm{~min}$ (Fig. 6).

To obtain a physiological measure of the reconstitution of tight junctions between newly apposed cells, electrical current pulses were passed across the epithelium while a pair of microelectrodes connected to a differential amplifier monitored the resultant potentials just above the epithelial surface $(9,10)$. Because more ionic current flows across an epithelium at regions of lower transepithelial resistance in such a recording configuration, greater potentials are recorded above sites of epithelial leakage. Immediately after the removal of a cell, a high leakage current was demonstrable at the site of the lesion (Figs. 1 and 2). This subsided gradually as contiguous cells advanced into the defect left by the missing cell, and reached control values by $30 \mathrm{~min}$ after damaging (Fig. 3). High-resolution experiments, which detected the normal slight leakage of current through tight junctions $(9,10)$, confirmed that transepithelial resistance at the site of removal of a cell reached control values after about $30 \mathrm{~min}$.

To corroborate the physiological findings, the sites of cell removal were examined by electron microscopy. Epithelia were fixed without damaging and at various stages of recovery from lesions by immersion for 50-90 min in a fixative consisting of $150 \mathrm{mM}$ glutaraldehyde, $800 \mathrm{mM}$ sodium cacodylate, and $5 \mathrm{mM}$ calcium chloride at $\mathrm{pH}$ 7.3. After fixation, specimens were washed in cacodylate buffer, postfixed in osmium tetroxide, stained en bloc with uranyl acetate, dehydrated in ethanol, and embedded in Epon. Thin sections stained with uranyl acetate and lead citrate were examined with a Philips EM 200 or EM 301 microscope.

Necturus gallbladder cells are normally joined together by typical tight junctions which appear in transmission electron micrographs as 5-10 points of membrane fusion (Fig. 8), and in freeze-fracture micrographs as a comparable number of anastomosing strands (Fig. 9). After a lesion, the surviving cells extended numerous microspike processes which met at the defect's center in roughly $15 \mathrm{~min}$. After 30 min of recovery, the cells were closely apposed to one another (Fig. 12), and their apical membranes showed multiple points of fusion characteristic of tight junctions (Figs. 10 and 11). These fusions, which were not always in their normal, ordered positions at cellular apices and which lacked the usual neat spacing of unperturbed tight junctions, nevertheless extended through multiple serial sections, and thus constituted at least segments of, if not complete, tight junctions.

If the recording scans or microscopical sectioning had been misoriented, normal intercellular contacts (rather than nascent ones) would have been the objects of study. To ex- 

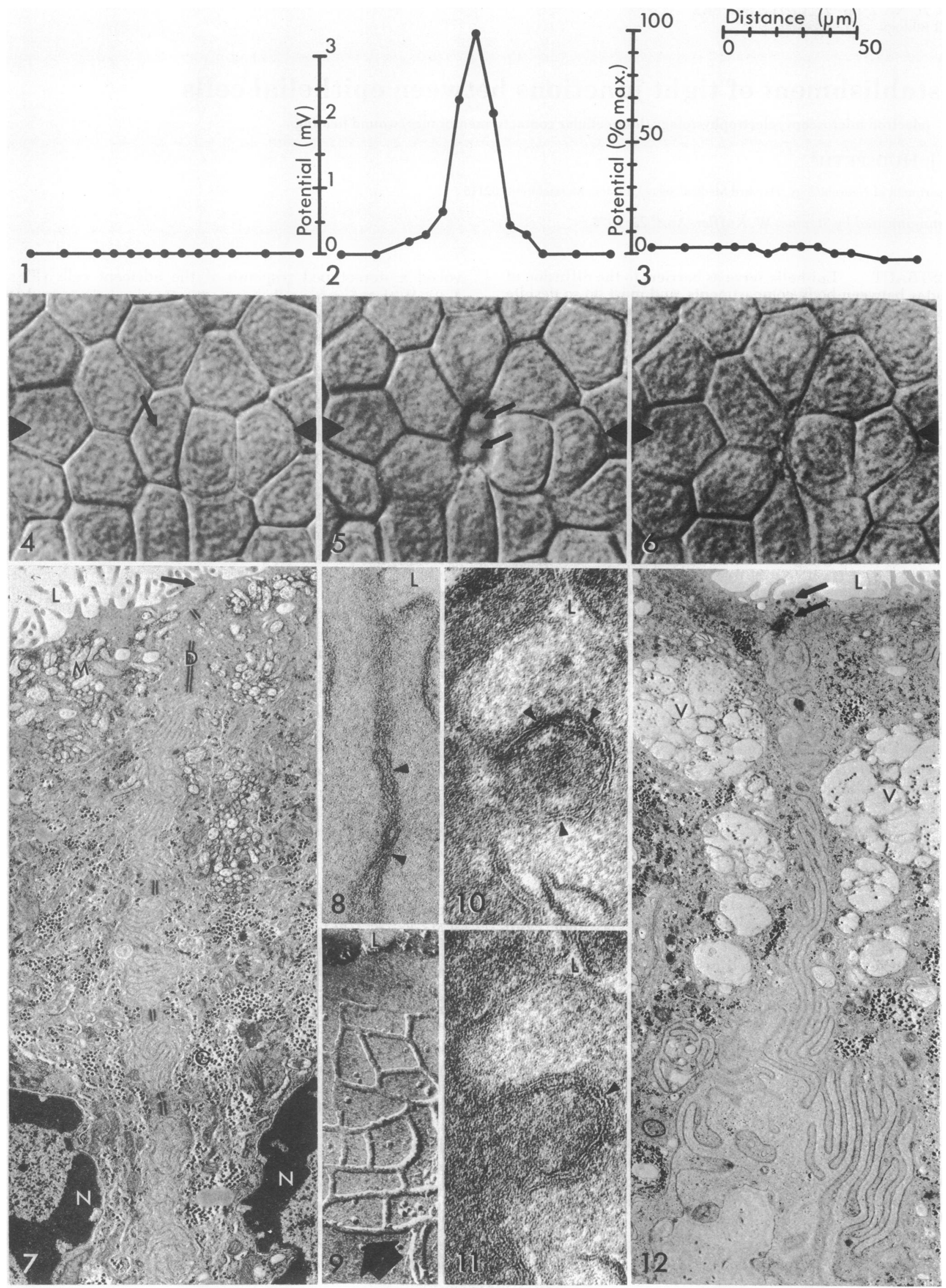

FIGS. 1-12. (Legend appears at top of following page.) 
FIGS. 1-3 (on preceding page). Scans of extracellular potentials produced by the transepithelial flow of a pulsed current of $5 \mu \mathrm{A} / \mathrm{mm}^{2}$. The measuring electrode was moved along the lines defined by arrowheads in Figs. 4-6, and measurements were taken at positions in register with the data points. An increase in the voltage signal indicates an enhanced transepithelial current flow, and hence a local decrease in transepithelial resistance. The ordinate scales show the voltage scans as measured potentials and as percentages of the maximum; the abscissal distance scale applies also to Figs. 4-6.

Figs. 4-6 (on preceding page). Nomarski light micrographs of Necturus gallbladder epithelial cells viewed in the living state from their apical surfaces with a $\times 40$, water-immersion lens. The intercellular borders are clearly demarcated. $\times 500$. Figs. 1 and 4 . A control preparation with homogeneous transepithelial current flow; the cell to be damaged is indicated (arrow). Figs. 2 and 5. Five min after the removal of a single cell, the surrounding cells are seen to be migrating into the epithelial defect, which contains two fragments of cytoplasm from the missing cell (arrows). The voltage trace shows a high transepithelial current in the region of the lacuna. Figs. 3 and 6 . Thirty min after the lesion, the remaining cells have closed the defect in the epithelium, and the voltage trace has returned to control levels, indicating a restoration of the normal transepithelial resistance.

FIGS. 7-12 (on preceding page). Electron micrographs of the regions of contact between cells of the Necturus gallbladder. In each case, the cellular apices are at the top of the picture, and the lumen is indicated (L). Fig. 7. A low-power view of the apical region of two cells shows their extensively convoluted boundary, which is punctuated with prominent desmosomes (D). The tight junction between the two cells occurs within the terminal bar (apical junctional complex) at the extreme apices of the cells (arrow). N, nucleus; M, mucous granules; G, glycogen particles. $\times 9500$. Fig. 8 . A high-power view of the normal tight junction between two such cells. The junction consists of multiple contacts between the two apposed membranes (arrowheads) with focal obliterations of the intercellular space. $\times 110,000$. Fig. 9. A freezefracture preparation of a normal intercellular junction demonstrating that the contact consists of six to eight highly interconnected strands which run circumferentially about the cells' apices. The broad arrow shows the direction of platinum shadowing. $\times 110,000$. Figs. 10 and 11 . High-power micrographs of apical contacts between two gallbladder cells $30 \mathrm{~min}$ after the removal of the cell initially separating them. The cytoplasm of one cell (on the left) is markedly dense, a frequent finding in such circumstances. The cells are joined by a tight junction characterized by fusion of the outer membrane leaflets and focal obliterations of the intercellular space (arrowheads). Some sites of membrane fusion have been traced for up to $0.5 \mu \mathrm{m}$ in this group of serial sections. $\times 110,000$. Fig. 12. A low-power view of two cells $30 \mathrm{~min}$ after the intervening cell was removed. The intercellular boundary is relatively normal, but has only a single desmosome. Both cells contain numerous large vacuoles (V) filled with phagocytized detritus from the missing cell. Newly established tight junctions, such as those of Figs. 10 and 11 , occur in the apical region between the arrows. $\times 9500$.

clude this unlikely possibility, experiments were done in which rows of 10 or more cells were removed. Current scanning and sectioning were then performed at a right angle to the linear wound and across its midpoint. The physiological and anatomical results confirmed those after single-cell lesions, and showed a similar timecourse of junctional reconstitution.

Physiologically functional and morphologically recognizable tight junctions can thus be established within about 30 min after a lesion, or within roughly $15 \mathrm{~min}$ of the approximation of the cells' plasma membranes. The latter time presumably represents an upper limit for the duration of junctional formation, because the cells involved are initially separated by a distance of about $30 \mu \mathrm{m}$, and since the lesioning process could be deleterious to surviving cells. Nonetheless, the results suggest that tight junctions, like gap junctions (11-13), can be established in the few minutes required for the maintenance of an epithelial permeability barrier in the face of rapid cell turnover. Repair of epithelial defects occurs with a similar timecourse in other organs of Necturus, including the stomach, large and small intestines, and urinary bladder (14). It remains to be determined whether new junctions are synthesized de novo, assembled from prefabricated components, or reconstituted from fragments of previously existing junctions.

I thank Drs. B. Forslind, E. A. Kravitz, U. J. McMahan, and T. N. Wiesel for the use of equipment, Dr. A. G. Yee for assistance in freeze-fracturing, and Drs. S. W. Kuffler, E. Raviola, and A. E. Stuart for comments on the manuscript. This research was supported in part by USPHS Grant MH-07084 and by a fellowship from the Alfred P. Sloan Foundation.

1. Farquhar, M. G. \& Palade, G. E. (1963) "Junctional complexes in various epithelia," J. Cell Biol. 17, 375-412.
2. Brightman, M. W. \& Reese, T. S. (1969) "Junctions between intimately apposed cell membranes in the vertebrate brain," J. Cell Biol. 40, 648-677.

3. Staehelin, L. A., Mukherjee, T. M. \& Williams, A. W. (1969) "Freeze-etch appearance of the tight junctions in the epithelium of small and large intestine of mice," Protoplasma 67, 165-184.

4. Staehelin, L. A. (1973) "Further observations on the fine structure of freeze-cleaved tight junctions," J. Cell Sci. 13, 763-786.

5. Friend, D. S. \& Gilula, N. B. (1972) "Variations in tight and gap junctions in mammalian tissues," J. Cell Biol. 53, 758776.

6. Claude, P. \& Goodenough, D. A. (1973) "Fracture faces of zonulae occludentes from 'tight' and 'leaky' epithelia," J. Cell Biol. 58, 390-400.

7. Leblond, C. P. \& Walker, B. E. (1956) "Renewal of cell populations," Physiol. Rev. 36, 255-276.

8. McMahan, U. J. \& Kuffler, S. W. (1971) “Visual identification of synaptic boutons on living ganglion cells and of varicosities in postganglionic axons in the heart of the frog," Proc. Roy. Soc. London Ser. B. 177, 485-508.

9. Frömter, E. \& Diamond, J. (1972) "Route of passive ion permeation in epithelia," Nature New Biol. 235, 9-13.

10. Frömter, E. (1972) "The route of passive ion movement through the eipthelium of Necturus gallbladder," J. Membr. Biol. 8, 259-301.

11. Loewenstein, W. R. (1967) "On the genesis of cellular communication," Dev. Biol. 15, 503-520.

12. Asada, Y. \& Bennett, M. V. L. (1971) "Experimental alteration of coupling resistance at an electrotonic synapse," J. Cell Biol. 49, 159-172.

13. Pappas, G. D., Asada, Y. \& Bennett, M. V. L. (1971) "Morphological correlates of increased coupling resistance at an electrotonic synapse," J. Cell Biol. 49, 173-188.

14. Hudspeth, A. J. (1973) in Intercellular Junctions in Epithelia, Ph.D. Dissertation, Harvard University, Cambridge, Mass., pp. 68-81. 